\title{
Unraveling the concealed and calculated cardiovascular risks in diabetes
}

\author{
Weerarathna $\mathbf{T} \mathbf{P}^{1}$ \\ Journal of the Ceylon College of Physicians, 2016, 47, 15-19
}

\begin{abstract}
Cardiovascular diseases (CVD) account for substantial morbidity and mortality in patients with type 2 diabetes (T2DM). Those with T2DM carry a three to four fold risk of developing CVD compared to healthy individuals. ${ }^{1}$ Statistics from developed counties reveal a significant decline in CVD mortality among people without diabetes. ${ }^{2}$ Despite intensified attempts to control risk factors, CVD mortality in diabetes has increased with a rising trend in the South Asian region. ${ }^{3}$ With the high incidence of diabetes manifested at a relatively younger age with greater adverse risk factor profiles favoring accelerated atherosclerosis, health authorities in the South Asian countries are facing several dilemmas in controlling CVD.
\end{abstract}

Epidemiological research highlights the pandemic of diabetes in Sri Lanka. ${ }^{4}$ In the midst of this pandemic, several new developments have taken place in this arena. Research on these new areas including diagnostic utility of glycated hemoglobin in patients with high CVD risk, performance of CVD prediction tools in therapeutic decision making and value of surrogate markers to detect subclinical atherosclerosis is sparse in the local setting. Applications of some of these modern tools are hampered by the paucity of locally relevant literature. This oration includes a series of clinical research carried out in these new arenas in order to unravel concealed and calculated CVD risks in patients with T2DM.

In the first study which is a clinical audit among patients with no previous history of diabetes presenting with acute coronary syndrome (ACS) we tested the value of glycated hemoglobin estimation in unraveling concealed glucose abnormalities. Subsequent studies are based on data obtained from patients screened at a Diabetes Centre in Southern Sri Lanka.

${ }^{1}$ Professor in Medicine, Faculty of Medicine, University of Ruhuna, Karapitiya, Sri Lanka.

E-mail: thilak.priyantha@yahoo.com
This database consisted of a diverse population of patients with T2DM including newly diagnosed and those with varying duration and already under treatment. All patients underwent clinical and laboratory tests recommended by the American Diabetes Association (ADA) for annual screening. Biochemical investigations were carried out in a single laboratory using standard protocols. These studies unraveled significant information on lipid abnormalities among newly diagnosed patients with type 2 diabetes; estimation of cardiovascular risk according to different risk estimation tools and correlation of the cardiovascular risk scores obtained using risk estimation tools with carotid intima media thickness, a clinical surrogate of atherosclerosis.

Concealed glucose abnormalities among patients with acute coronary syndromes

To estimate the proportion of patients with concealed abnormalities in glucose, namely prediabetes and diabetes, among patients with no known diabetes admitted with ACS, the fasting blood sugar and $\mathrm{HbA} 1 \mathrm{c}$ were estimated in 125 patients admitted to a tertiary care hospital.

Results revealed that according to $\mathrm{HbA} 1 \mathrm{c}, 47 \%$ (56) and 53\% (69) patients with ACS had diabetes and pre diabetes respectively. The corresponding percentages in each category according to fasting blood glucose testing were (6) $4 \%$ and (20) $20 \%$ (Table1). There were no significant differences in the mean age, body mass index or waist circumference of patients in the categories with no diabetes on both testing methods and diabetes only on HbA1c.

Table 1. Number of ACS patients in different glycemic categories

\begin{tabular}{lcc}
\hline & FBS $\mathrm{mg} / \mathrm{dl}$ & HbA1c \% \\
\hline Normal & 99 & None \\
IFG/Pre-diabetes & 20 & 69 \\
Diabetes & 6 & 56 \\
\hline
\end{tabular}


This audit revealed that based on $\mathrm{HbA1c}$, out of 125 patients almost every patient with ACS has either diabetes or pre diabetes. Compared to fasting blood glucose alone, additional testing for $\mathrm{HbA1c}$ in patients with ACS increased the number of patients with newly diagnosed diabetes by tenfold and pre diabetes by two and a half fold.

The prevalence of glucose abnormalities among patients without previous history of diabetes presenting with ACS in other studies vary between $21 \%-84 \%$. A study conducted in India revealed that $84 \%$ of patients with ACS with no history of diabetes have abnormalities in glucose metabolism based on glucose tolerance test (OGTT). ${ }^{5}$ Using HbA1c cut-off level of $6.5 \%$, the prevalence of diabetes in that study was $25 \%$.

\section{Associations of adverse CVD risk profile in newly diagnosed diabetes}

Recognition of patterns and associations of modifiable CVD risk profile at the time of diagnosis of diabetes would enable the adoption of more intensive primary prevention strategies to reduce CVD morbidity and mortality.

In a cross sectional study, among patients with newly diagnosed diabetes included in the database, the prevalence of abnormal values of low density lipoproteins (LDL), triglycerides (TG), high density lipoproteins (HDL) and systolic and diastolic blood pressure (SBP and DBP) in 412 patients was estimated. Each risk factor was determined according to the ADA criteria. Associations of each risk factor (dependent variable) with gender, age at onset of diabetes, waist circumference (WC) and body mass index (BMI) (independent variables) were studied using logistic regression.

Males accounted for $71 \%$ of the sample. Mean (SD) age, and age at onset of diabetes were 49(11) and $44(11)$ years. LDL > $100 \mathrm{mg}$ was prevalent in $81 \%$ and low HDL and TG $>150 \mathrm{mg} / \mathrm{dL}$ levels were detected in $17.1 \%, 18.5 \%$. SBP > $140 \mathrm{~mm} \mathrm{Hg}$ and $\mathrm{BP}>90 \mathrm{~mm}$ $\mathrm{Hg}$ were prevalent in $15 \%$ and $12 \%$. Regression analysis revealed significant associations of male gender OR 7.98 ( 95\% Cl 4.39 - 14.49) and younger age at onset of diabetes (OR $0.9595 \% \mathrm{Cl} 0.93-0.98$ ) with lower HDL (Table 2).

This study unraveled a high prevalence (81.2\%) of adverse levels of LDL (over $100 \mathrm{mg} / \mathrm{dL}$ ) at the time of diagnosis of diabetes with no significant gender difference. Male gender was associated with significantly higher odds of having a lower HDL level. Of the clinical parameters, younger age at onset of diabetes revealed a significant association with higher TG and lower HDL levels. BMI revealed a positive association with SBP, TG and low HDL but a statistically significant association of BMI was only seen with lower HDL level.

Studies conducted in multi ethnic settings have revealed ethnic variations in the prevalence of CVD risk factors in diabetes. ${ }^{6}$ A meta-analysis of 24,000 subjects reported that Asian Indians with and without diabetes compared to Caucasians and Chinese have significantly higher odds of lower HDL and higher TG levels. ${ }^{7}$ They also reported significantly lower HDL in males compared to females of Asian Indian ethnicity. A study conducted in multiethnic population in the United Kingdom revealed comparatively worse lipid profiles with lower HDL levels and higher CVD event

Table 2. Association of CVD risk factors with age, gender and anthropometry among newly diagnosed Type 2 diabetics

\begin{tabular}{|c|c|c|c|c|c|c|c|c|c|c|}
\hline \multirow[b]{2}{*}{$\begin{array}{l}\text { Clinical } \\
\text { Variable }\end{array}$} & \multicolumn{2}{|c|}{ LDL } & \multicolumn{2}{|c|}{ HDL } & \multicolumn{2}{|c|}{ TG } & \multicolumn{2}{|c|}{ SBP } & \multicolumn{2}{|c|}{ DBP } \\
\hline & $\begin{array}{l}\text { Odds ratio } \\
(95 \% \mathrm{Cl})\end{array}$ & $\begin{array}{c}P \\
\text { Value }\end{array}$ & $\begin{array}{l}\text { Odds ratio } \\
(95 \% \mathrm{Cl})\end{array}$ & $\begin{array}{c}P \\
\text { Value }\end{array}$ & $\begin{array}{l}\text { Odds ratio } \\
(95 \% \mathrm{Cl})\end{array}$ & $\begin{array}{c}P \\
\text { Value }\end{array}$ & $\begin{array}{l}\text { Odds ratio } \\
(95 \% \mathrm{Cl})\end{array}$ & $\begin{array}{c}P \\
\text { Value }\end{array}$ & $\begin{array}{l}\text { Odds ratio } \\
(95 \% \mathrm{Cl})\end{array}$ & $\begin{array}{c}P \\
\text { Value }\end{array}$ \\
\hline $\begin{array}{l}\text { Age at } \\
\text { diagnosis }\end{array}$ & $\begin{array}{c}1.003 \\
(0.98-1.03)\end{array}$ & 0.84 & $\begin{array}{c}0.95 \\
(0.93-0.98)\end{array}$ & $<0.01$ & $\begin{array}{c}0.95 \\
(0.93-0.98)\end{array}$ & $<0.01$ & $\begin{array}{c}1.09 \\
(1.05-1.13)\end{array}$ & $<0.01$ & $\begin{array}{c}1.062 \\
(1.006-1.121)\end{array}$ & 0.03 \\
\hline $\begin{array}{l}\text { Gender } \\
\text { (Male) }\end{array}$ & $\begin{array}{c}2.46 \\
(1.19-5.07)\end{array}$ & 0.15 & $\begin{array}{c}7.98 \\
(4.39-14.49)\end{array}$ & $<0.01$ & $\begin{array}{c}0.73 \\
(0.39-1.36)\end{array}$ & 0.32 & $\begin{array}{c}1.16 \\
(0.53-2.55)\end{array}$ & 0.71 & $\begin{array}{c}1.28 \\
(0.39-4.11)\end{array}$ & 0.68 \\
\hline WC & $\begin{array}{c}0.98 \\
(0.93-1.03)\end{array}$ & 0.4 & $\begin{array}{c}0.95 \\
(0.91-0.99)\end{array}$ & 0.03 & $\begin{array}{c}1.02 \\
(0.98-1.07)\end{array}$ & 0.36 & $\begin{array}{c}0.98 \\
(0.92-1.04)\end{array}$ & 0.53 & $\begin{array}{c}1.01 \\
(0.92-1.11)\end{array}$ & 0.86 \\
\hline BMI & $\begin{array}{c}0.99 \\
(0.87-1.12)\end{array}$ & 0.86 & $\begin{array}{c}1.139 \\
(1.07-1.28)\end{array}$ & 0.04 & $\begin{array}{c}1.003 \\
(0.88-1.13)\end{array}$ & 0.96 & $\begin{array}{c}1.02 \\
(0.86-1.20)\end{array}$ & 0.82 & $\begin{array}{c}0.98 \\
(0.76-1.25)\end{array}$ & 0.86 \\
\hline
\end{tabular}


rate among the South Asians compared to Caucasians. ${ }^{8}$ A study conducted in Singapore among T2DM patients from different Asian ethnicities have reported that Asian Indians have significantly lower HDL cholesterol levels compared to Malays and Chinese patients. ${ }^{9}$ Previous study of 592 patients with newly diagnosed T2DM conducted in Sri Lanka reported similar prevalence of high TG (14\%) and low HDL (12\%) but they do not report on the prevalence of high LDL. ${ }^{10}$ High TG level and low HDL cholesterol level is a surrogate marker of insulin resistance and is also considered as the hallmark of atherogenic dyslipidemia. ${ }^{11}$

\section{Cardiovascular risk estimation tools in type 2 diabetes}

Modification of CVD risk factors is the major pathway followed to reduce increased CVD morbidity and mortality in patients with T2DM. Two approaches are used to select patients for CVD risk factor modification. The population based total cardiovascular risk assessment approach recommended by the World Health Organization (WHO) uses five easily measurable CVD risk factors (total cholesterol, SBP, age, gender, smoking status). ${ }^{12}$ The risk estimate thus obtained $(<10 \%=$ low, $10-20=$ moderate, $>20 \%=$ high) is used for decision making to start lipid lowering and antihypertensive therapy. This approach excludes more important CVD risk factors (LDL, DBP) which need participation of medical personnel and involves laboratory testing for accurate estimation. However in the target based approach, more emphasis is focused on interventions to attain recommended therapeutic targets for LDL and DBP; as there is large body of evidence of clinical benefits from achieving the recommended therapeutic targets.

In this background, the Ministry of Health in Sri Lanka adopted WHO total CVD risk assessment approach to select individuals for lipid lowering and antihypertensive therapy in primary care. In the study 3 , we analyzed effectiveness of WHO approach to detect patients with adverse LDL and DBP in to its high risk $(>20 \%)$ category. In this study, we compared WHO approach with another CVD risk assessment tool; Framingham risk score (FRS) which in addition to parameters in WHO tool, uses HDL cholesterol as well. As HDL is significantly low among males and patients with younger onset T2DM, we assumed FRS could select higher number of patients with adverse CVD risk profile in to its high risk category than the WHO tool.

\section{Application of CVD risk estimation tools in the Sri Lankan setting}

We calculated CVD risk scores of newly diagnosed patients with T2DM included in the database using WHO model. In the category with low $(<20 \%)$
CVD risk score, proportions with low density lipoproteins $(\mathrm{LDL})>101 \mathrm{mg} / \mathrm{d} L$ and diastolic blood pressure $(\mathrm{DBP})>91 \mathrm{mmHg}$, triglycerides $(\mathrm{TG})>151 \mathrm{mg} / \mathrm{dL}$ were estimated. Sensitivity, positive (PPV) and negative (NPV) predictive values of LDL, TG and DBP above the recommended value against WHO risk cut-off of $20 \%$ were calculated.

Results revealed that $97.6 \%$ of the study sample was categorized into low risk category. The percentages of subjects in the low risk category having LDL, DBP and TG levels above the recommended level were $82.4 \%, 2.1 \%$ and $16.3 \%$ respectively. Sensitivity of WHO risk level of $20 \%$ in detecting high LDL, DBP, TG and low HDL were 2.9\%, 36.3\%, 1.85\%. PPV and NPV for the same parameters were $100 \%$ vs. $17.59 \%$, $50 \%$ vs. $97.8 \%$ and $12.5 \%$ vs. $83.3 \%$ respectively.

Findings of this study highlighted the poor performance of WHO total CVD risk assessment tool in detecting individuals with one of the most important CVD risk factors, LDL cholesterol > $101 \mathrm{mg} / \mathrm{dl}$ into the high risk category. Out of the interventions to reduce adverse CVD outcomes, LDL reduction with statin therapy has proven very safe and cost effective.

Exclusion of a substantial majority needing LDL reduction by the WHO approach raises many concerns. We recommend further testing of this tool before its widespread use or adoption of more sensitive CVD risk assessment method in primary care to select patients for CVD risk factor modification.

\section{Comparison of two CVD risk estimation tools in therapeutic decision making}

WHO cardiovascular disease risk score and Framingham risk scores (FRS) are used to estimate total cardiovascular risk (TCVDR) to guide therapeutic decisions. We estimated percentages of newly diagnosed diabetics with LDL and DBP above recommended level who qualify for therapy at $>20 \%$ cut-points of each TCVDR scores.

We calculated individual TCVDR using FRS and WHO scores in 331 patients and categorized them into five CVD risk categories $(1=<10 \%, 2=10-20 \%$, $3=21-30 \%, 4=31-40 \%, 5=>41 \%)$. Percentages with LDL > $101 \mathrm{mg} / \mathrm{dL}$ and DBP >91 $\mathrm{mm} \mathrm{Hg}$ in each risk category were estimated.

We found that $277 / 331$ had LDL $>101 \mathrm{mg} / \mathrm{dl}$ and WHO categories 1-5 included $90.61 \%, 6.13 \%, 1.44 \%$, $1.08 \%, 0.72 \%$ of them and the corresponding figures with FRS were 48.01\%, 38.9\%, 11.55\%, 1.44\%, 1.04\% respectively. 43/ 331 of sample had DBP > $91 \mathrm{~mm} \mathrm{Hg}$ and percentages in the corresponding $\mathrm{WHO}$ and FRS risk categories were 55.81\%, 25.58\%, 6.97\%,6.97\% $4.65 \%$ and $9.3 \%, 44.18 \%, 39.53 \%, 0$, and $6.97 \%$ respectively. 
This study revealed that both risk estimation tools fail to detect the majority of diabetic patients with LDL and DBP needing treatment in primary care. Using $>20 \%$ CVD risk level of WHO and FRS tools, 95\% and $86 \%$ with adverse LDL and $62 \%$ and $32 \%$ with high DBP above level were deprived of lipid lowering and antihypertensive drug therapy respectively.

Comparison performances of two total cardiovascular risk assessment tools unravel their relative lack of sensitivity to detect diabetic patients with adverse levels of the most important CVD risk factor, LDL cholesterol. WHO risk assessment tool is developed to help health authorities in low and middle income countries to guide decision making in primary care on lipid lowering and antihypertensive therapy. They have used easily measurable parameters which can be measured even by a nurse or para medical worker. We have found that the large majority of Sri Lankan patients have high LDL and some; especially the younger patients have low HDL levels. Detection of both these needs a fasting lipid profile. Sri Lanka is gradually becoming a middle and high income country. Measurement of DBP and estimation of lipid profile can be carried out without much cost. Generic statins are available at a relatively low price. Therefore, denying statin therapy by excluding more than $90 \%$ with LDL level more than $101 \mathrm{mg} / \mathrm{Dl}$ at the time of diagnosis of diabetes in primary care level can be counterproductive. Findings of study 2, 3 and 4 unravel the need of persistence with target based approach to modify CVD risk factors until more sensitive tools which are validated in local setting are introduced.

\section{Association of surrogate of atherosclerosis with CVD risk estimates}

Atherosclerosis underlies all, if not most clinical forms of CVD. Atherosclerosis is a concealed process that progress over decades and there are non-invasive tools to detect subclinical stages atherosclerosis before clinical manifestations. Measurement of carotid intima media thickness (CIMT) by ultrasound is a noninvasive method to assess atherosclerosis. CIMT is used as a surrogate marker to guide the need of more invasive testing for CVD. ${ }^{13}$ There is limited data on the use of CIMT in Sri Lankan patients.

We studied the associations between CIMT and CVD risk estimates obtained by three different tools in seventy randomly selected patients with T2DM from our database. For this study in addition to WHO and FRS risk estimation tools we also used the risk engine developed after the United Kingdom Prospective Diabetes Study (UKPDS risk engine).

CIMT was measured in 70 randomly selected subjects who underwent annual screening for complications of diabetes in the Diabetes Centre. CVD risk score was estimated using the $\mathrm{WHO}, \mathrm{FRS}$ and UKPDS risk engines. Association between the CVD risk estimates with each tool with CIMT was studied with Pearson correlation.

Thirty six $(51.4 \%)$ of the study subjects were males. Mean age was 56 years $(S D=10)$, mean duration of diabetes was 10 years $(S D=8)$ and mean CIMT was 0.886 (SD=0.293). Correlation coefficient ( $r$ ) between CIMT and UKPDS, FRS and WHO risk estimates were $+0.26(p=0.03),+0.2(p=0.2),-0.06$ $(p=0.57)$ respectively. Results of this study revealed that out of the three risk estimates both the UKPDS and FRS CVD risk scores were positively and significantly associated with CIMT and the WHO risk estimate has a negative association highlighting its relative lack of sensitivity to detect underlying atherosclerosis.

Some studies have revealed that the duration of diabetes and several lipid parameters, including low $\mathrm{HDL}$, are significantly associated with CIMT. ${ }^{14}$ Failure to find such associations in our study could be due to smaller sample size. Nevertheless, finding that a T2DM patient with a higher CVD risk score is more likely to have subclinical atherosclerosis as manifested by CIMT is significant. Calculated CVD risk score with UKPDS or FRS risk estimation tools in a Sri Lankan patient with T2DM can unravel concealed atherosclerosis in asymptomatic stages.

\section{Summary and conclusions}

From the series of clinical research included in this oration, several concealed and calculated risks in CVD could be unraveled.

1. HbA1c test in patients with ACS can unravel diabetes which is concealed by testing with FBS alone.

2. More atherogenic lipid profile is a significantly concealed CVD risk in younger males at the time of diagnosis of T2DM.

3. Calculated CVD risk assessment with total cardiovascular risk assessment tool recommended by the WHO fails to recognize the majority with adverse LDL and DBP and requiring lipid lowering and antihypertensive therapy.

4. Framingham Risk Score is marginally more sensitive, yet excludes the majority with adverse LDL needing therapy.

5. Concealed atherosclerosis manifested as CIMT is reliably predicted in Sri Lankan subjects with T2DM with the calculated UKPDS risk score. 


\section{References}

1. Marks JB and Raskin P. Cardiovascular risk in diabetes: a brief review. J Diabetes Complications 2000; 14: 108-15.

2. Baldwin MD. Assessing cardiovascular risk factors and selecting agents to successfully treat patients with type 2 diabetes mellitus. The Journal of the American Osteopathic Association 2011; 111: S2-12.

3. Gholap N, Davies M, Patel K, Sattar N, Khunti K. Type 2 diabetes and cardiovascular disease in South Asians. Prim Care Diabetes 2011; 5: 45-56.

4. Pinidiyapathirage MJ, Kasturiratne A, Ranawaka UK, et al. The burden of diabetes mellitus and impaired fasting glucose in an urban population of Sri Lanka. Diabet Med 2013; 30: 326-32.

5. Ramachandran A, Chamukuttan S, Immaneni S, et al. High incidence of glucose intolerance in Asian-Indian subjects with acute coronary syndrome. Diabetes Care 2005; 28: 2492-6.

6. Davis TM, Cull CA and Holman RR. Relationship between ethnicity and glycemic control, lipid profiles, and blood pressure during the first 9 years of type 2 diabetes: U.K. Prospective Diabetes Study (UKPDS 55). Diabetes Care 2001; 24: 1167-74.

7. Zhang L, Qiao Q, Tuomilehto J, et al. Distinct ethnic differences in lipid profiles across glucose categories. $J$ Clin Endocrinol Metab 2010; 95: 1793-801.

8. Bellary S, O'Hare JP, Raymond NT, et al. Premature cardiovascular events and mortality in south Asians with type 2 diabetes in the United Kingdom Asian Diabetes Study - effect of ethnicity on risk. Curr Med Res Opin. 2010; 26: 1873-9.

9. Hughes K, Yeo PP, Lun KC, et al. Cardiovascular diseases in Chinese, Malays, and Indians in Singapore. II. Differences in risk factor levels. Journal of Epidemiology and Community Health 1990; 44: 29-35.

10. Weerasuriya N, Siribaddana S, Dissanayake A, Subasinghe Z, Wariyapola D, Fernando DJ. Long-term complications in newly diagnosed Sri Lankan patients with type 2 diabetes mellitus. QJM: monthly Journal of the Association of Physicians 1998; 91: 439-43.

11. Eeg-Olofsson K, Gudbjornsdottir S, Eliasson B, Zethelius B, Cederholm J. The triglycerides-to-HDL-cholesterol ratio and cardiovascular disease risk in obese patients with type 2 diabetes: An observational study from the Swedish National Diabetes Register (NDR). Diabetes Res Clin Pract. 2014.

12. Otgontuya D, Oum S, Buckley BS and Bonita R. Assessment of total cardiovascular risk using $\mathrm{WHO} / \mathrm{ISH}$ risk prediction charts in three low and middle income countries in Asia. BMC Public Health. 2013; 13: 539.

13. Rubinat E, Marsal JR, Vidal T, et al. Subclinical Carotid Atherosclerosis in Asymptomatic Subjects With Type 2 Diabetes Mellitus. The Journal of Cardiovascular Nursing. 2015.

14. Irie $\mathrm{Y}$, Katakami $\mathrm{N}$, Kaneto $\mathrm{H}$, et al. The risk factors associated with ultrasonic tissue characterization of carotid plaque in type 2 diabetic patients. J Diabetes Complications 2014; 28: 523-7. 